INPLASY

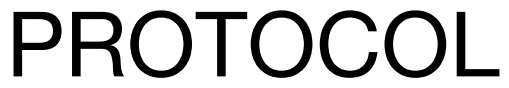

To cite: Cao et al. Efficacy and Safety of Acupuncture for Postpartum depression:a protocol for systematic review and network meta-analysis. Inplasy protocol 2020120053. doi:

10.37766/inplasy2020.12.0053

Received: 09 December 2020

Published: 09 December 2020

Corresponding author:

Cao Yue

942133430@qq.com

Author Affiliation:

Chengdu University of TCM

Support: SQ2018YFC170240.

Review Stage at time of this submission: The review has not yet started.

Conflicts of interest: None.

\section{Efficacy and Safety of Acupuncture for Postpartum depression: a protocol for systematic review and network meta-analysis}

Cao, Y1; Yuan, J2; Cao, W3; Wen, CB4.

Review question / Objective: P: Postpartum depression patients I:The treatment group included all kinds of acupuncture therapy, such as simple acupuncture, electroacupuncture, auricular point or combined with other treatments. C: the control group was treated with blank control group or western medicine alone. Western drugs included antidepressants: serotonin reuptake inhibitors SSRIs (fluoxetine, citalopram, paroxetine and sertraline), norepinephrine reuptake inhibitors (venfaraxine, mirtazapine, duloxetine), and antianxiety drugs: benzodiazepines (diazepam, lorazepam, clonazepam, alprazolam, estazolam). Non-benzodiazepine (buspirone, Deanxin), etc. O:Score of Hamilton Depression scale ((Hamilton Depression Scale,HAMD); score of Edinburgh Postpartum Depression scale (Edinburgh "postnatal" depression "scale,EPDS"); Total clinical effective rate: clinical efficacy was evaluated according to HAMD or EPDS score reduction rate, reduction rate $=[$ (pre-treatment score-post-treatment score $) /$ total pretreatment score] $\times 100 \%$; recovery: score reduction rate $\geq$ $\mathbf{7 5} \%$; markedly effective: $\mathbf{5 0 \%} \leq$ score reduction rate $<\mathbf{7 5 \%}$; effective: $\mathbf{2 5 \%} \leq$ score reduction rate $<50 \%$. Invalid: the score reduction rate is less than $25 \%$.

INPLASY registration number: This protocol was registered with the International Platform of Registered Systematic Review and Meta-Analysis Protocols (INPLASY) on 09 December 2020 and was last updated on 09 December 2020 (registration number INPLASY2020120053).

\section{INTRODUCTION}

Review question / Objective: P: Postpartum depression patients I:The treatment group included all kinds of acupuncture therapy, such as simple acupuncture, electroacupuncture, auricular point or combined with other treatments. C: the control group was treated with blank control group or western medicine alone. Western drugs included antidepressants: serotonin reuptake inhibitors SSRIs 
(fluoxetine, citalopram, paroxetine and sertraline), norepinephrine reuptake inhibitors (venfaraxine, mirtazapine, duloxetine), and antianxiety drugs: benzodiazepines (diazepam, lorazepam, clonazepam, alprazolam, estazolam). Nonbenzodiazepine (buspirone, Deanxin), etc. O:Score of Hamilton Depression scale ((Hamilton Depression Scale,HAMD); score of Edinburgh Postpartum Depression scale (Edinburgh "postnatal" depression "scale,EPDS"); Total clinical effective rate: clinical efficacy was evaluated according to HAMD or EPDS score reduction rate, reduction rate $=$ [(pre-treatment scorepost-treatment score) / total pre-treatment score] $\times 100 \%$; recovery: score reduction rate $\geq 75 \%$; markedly effective: $50 \% \leq$ score reduction rate $<75 \%$; effective: $25 \%$ $\leq$ score reduction rate $<50 \%$. Invalid: the score reduction rate is less than $25 \%$.

Condition being studied: At present, the main treatment measures for postpartum depression are: drug therapy, psychotherapy and other treatments. Drugs include the selective serotonin uptake inhibitor (SSRIs) and other antidepressants or sedative hypnotics. Psychotherapy includes interpersonal psychotherapy ((IPT)) and cognitive behavioral therapy ((CBT),). Other treatments such as exercise therapy, repetitive transcranial magnetic stimulation ((rTMS)) and phototherapy, etc., but there is no clear conclusion on the effectiveness of different drug regimens. Acupuncture, as a green treatment, is widely used in the treatment of PPD, and a 2016 Meta analysis shows that acupuncture is equivalent to basic drugs and placebo in the treatment of postpartum depression. Most acupuncture studies on PPD usually choose positive drugs or placebos as the control group, lack of comparison between different acupuncture treatments. In order to meet the needs of comparing these different acupuncture interventions, we carried out Bayesian network Meta analysis to compare the advantages and disadvantages of two or more different acupuncture interventions, so as to provide clinicians with better decision-making.

\section{METHODS}

Participant or population: Postpartum depression patients.

Intervention: The treatment group included all kinds of acupuncture therapy, such as simple acupuncture, electroacupuncture, auricular point or combined with other treatments.

Comparator: The control group was treated with blank control group or western medicine alone. Western drugs included antidepressants: serotonin reuptake inhibitors SSRIs (fluoxetine, citalopram, paroxetine and sertraline), norepinephrine reuptake inhibitors (venfaraxine, mirtazapine, duloxetine), and antianxiety drugs: benzodiazepines (diazepam, lorazepam, clonazepam, alprazolam, estazolam). Non-benzodiazepine (buspirone, Deanxin), etc.

Study designs to be included: Randomized controlled trials (RCTs).

Eligibility criteria: The study included all RCT, associated with acupuncture interventions for the treatment of PPD, regardless of blindness and concealment. However, The written language is limited in English or Chinese.

Information sources: Our study will search the following electronic bibliographic databases: PubMed, EMBASE,The Cochrane Library, Scopus, Web of Science, China National knowledge Infrastructure (CNKI), Chongqing VIP Database, Wanfang Database and Chinese Biomedical Literature Database to find acupuncture interventions for related randomized controlled trials (RCT) for PPD.

Main outcome(s): Score of Hamilton Depression scale ((Hamilton Depression Scale,HAMD); score of Edinburgh Postpartum Depression scale (Edinburgh "postnatal" depression "scale,EPDS"); Total clinical effective rate: clinical efficacy was evaluated according to HAMD or EPDS score reduction rate, reduction rate $=[($ pretreatment score-post-treatment score) / 
total pre-treatment score] $\times 100 \%$; recovery: score reduction rate $\geq 75 \%$; markedly effective: $\mathbf{5 0 \%} \leq$ score reduction rate < $75 \%$; effective: $25 \% \leq$ score reduction rate $<50 \%$. Invalid: the score reduction rate is less than $25 \%$.

Quality assessment / Risk of bias analysis: According to the Cochrane Handbook, two independent reviewers(CW and $\mathrm{YJ}$ ) will evaluate the risk of bias in the study, including the generation of random sequences, the concealment of assignments, the blindness of participants, personnel and outcome evaluators, incomplete outcome data, selective reports, and other sources of bias. We divide each area into three levels: high risk of bias, unclear or low. Differences of opinion among reviewers will be resolved through discussion or negotiation with the third reviewer(CY).

Strategy of data synthesis: We will conduct a traditional meta-analysis for direct comparison. The influence of continuous variable data will be calculated by standardized mean difference (SMD), while the impact of binary variable data will be calculated by risk ratio (RR). The 95\% confidence interval (CI) of SMD and RR will also be calculated $(95 \% \mathrm{Cl})$. For indirect comparisons, reticular meta analysis is needed to mix the results to improve statistical efficiency. The network metaanalysis will be carried out using the "netmeta" package of the $R$ software. the results of direct and indirect comparisons will be presented in the form of a network diagram.

Subgroup analysis: We will conduct a subgroup analysis to search for potential inconsistencies and heterogeneity, such as depressive level, gender, ethnicity, etc.

Sensibility analysis: We will conduct a sensitivity analysis to verify the robustness of the results. Studies with uncertain or high-risk bias risks will be excluded to check whether the results will change.

Language: The written language is limited in English or Chinese.
Country(ies) involved: China.

Keywords: acupuncture; postpartum depression (PPD) ; Meta analysis.

Contributions of each author:

Author 1 - Cao Yue.

Author 2 - Yuan Jie.

Author 3 - Cao Wei.

Author 4 - Wen ChuanBiao. 